\title{
Verstößt die berufsrechtliche Regelung über die Teilgemeinschaftspraxis gegen die Berufsfreiheit?
}

Urteil des BGH vom 15.05.2014, Az.: I ZR 137/12

\section{Einleitung}

Die Möglichkeit des Zusammenschlusses von Ärzten in Teilgemeinschaftspraxen wurde auf dem 107. Deutschen Ärztetag 2004 in Bremen durch die beschlossene Neufassung der (Muster-) Berufsordnung (MBO) und die nachfolgende Übernahme in den meisten Berufsordnungen der Landesärztekammern eingeführt. Im Vertragsarztrecht erfolgte die Einführung der Teilgemeinschaftspraxis durch das Vertragsarztrechtsänderungsgesetz (VÄndG) vom 22.12.2006, welches am 01.01.2007 in Kraft getreten ist. Neben der Möglichkeit überörtliche Berufsausübungsgemeinschaften zu gründen und mehreren Berufsausübungsgemeinschaften anzugehören, wurde daneben als wesentliche weitere Liberalisierung eingeführt, dass Gemeinschaftspraxen nicht das gesamte Leistungsspektrum umfassen müssen.

Gerade im Bereich überweisungsabhängiger Fachgebiete wirft die Ausgestaltung der Teilgemeinschaftspraxis jedoch erhebliche Fragestellungen auf, da sie nicht dazu missbraucht werden soll, berufsrechtlich unzulässige Zuweisermodelle mit dem Rechtsschein zulässiger Berufsausübung zu versehen. Der Zuweisungscharakter von Zahlungen an Fachärzte, die ihre Patientenzuweisungen an die überweisungsgebundenen Fachgebiete von Rückvergütungen abhängig machen, soll nicht dadurch geheilt werden können, dass die Zuweiser diese Zahlungen nun als Gesellschafter über die Gewinnausschüttung einer formalrechtlich zulässigen Teilgemeinschaftspraxis erhalten. Mit anderen Worten soll die Vergesellschaftung von finanziellen Zuwendungen auch im Rahmen einer Teilgemeinschaftspraxis nicht möglich sein. Dabei wird allerdings häufig übersehen, dass diese Form der Zusammenarbeit auch im Rahmen einer regulären Berufsausübungsgemeinschaft vereinbart werden kann, ohne dass die beteiligten Ärzte mit entsprechenden Sanktionen rechnen müssen. Auch stellt die gesellschaftsrechtliche Beteiligung von Vertragsärzten als Gründer eines MVZ nach $\S$
95 Abs. 1a Satz 1 SGB V eine vom Gesetzgeber ausdrücklich gebilligte Möglichkeit einer Gewinnausschüttung an Ärzte dar, die dort keine vertragsärztliche Tätigkeit erbracht haben.

In der vorliegenden Entscheidung hat sich der BGH mit den berufsrechtlichen Vorgaben für die Beteiligung von Radiologen und anderen methodendefinierten Fachgebieten an einer Teilgemeinschaftspraxis auseinandergesetzt und ist zu dem Ergebnis gekommen, dass die Regelung in $\S 18$ Abs. 1 Satz 3 der Berufsordnung der Landesärztekammer Baden-Württemberg (BO BW) in Teilen gegen das Grundrecht der Ärzte auf Berufsausübungsfreiheit aus Art. 12 Abs. 1 GG verstößt.

\section{Sachverhalt \\ $\nabla$}

In Baden-Württemberg hatten sich 30 Fachärzte zu einer Partnerschaftsgesellschaft zusammengeschlossen, zu der 4 Radiologen gehörten. Gemäß § 2 Nr. 1 des Partnerschaftsvertrags hatten sich die Ärzte neben ihrer bisherigen Praxis zusätzlich zur standortübergreifenden Erbringung privatärztlicher Leistungen verbunden. Die Leistungserbringung sollte durch die Ärzte nach den jeweiligen Normen der privatärztlichen Abrechnungen erfolgen, wobei alle dem jeweiligen Fachgebiet vorbehaltenen privatärztlichen Leistungen einen gemeinsamen Leistungsinhalt der Teilgemeinschaftspraxis darstellten und die Leistungen im Namen der Gesellschaft abgerechnet werden sollten. Die Gewinnverteilung erfolgte in Höhe von 1\% vorab nach Köpfen. Die weiteren $99 \%$ wurden nach dem persönlich erbrachten Leistungsanteil des jeweiligen behandelnden Arztes ausgeschüttet.

Die Zentrale zur Bekämpfung unlauteren Wettbewerbs erhob Unterlassungsklage gegen die Partnerschaftsgesellschaft, um die gerichtliche Untersagung der Beteiligung der Radiologen in der Teilgemeinschaftspraxis zu erreichen. Die Klägerin hält die Beteiligung der Radiologen an der
Teilgemeinschaftspraxis für unzulässig, weil sie der Umgehung des $\S 31$ BO BW diene, wonach Ärzte für die Zuweisung von Patienten weder Vorteile gewähren noch sich versprechen lassen dürfen.

Die Klage wurde vom LG Mosbach mit der Begründung abgewiesen, dass das generelle Verbot der Zusammenarbeit mit Radiologen gemäß § 18 Abs. 1 Satz 3 Fall 1 BO BW verfassungswidrig sei. Die gegen dieses Urteil eingelegte Berufung der Klägerin vor dem OLG Karlsruhe war dagegen erfolgreich. Nach Ansicht des OLG Karlsruhe ist das berufsrechtliche Verbot gemäß § 18 Abs. 1 Satz 3 Fall 1 MBO zulässig und verhältnismäßig. Gegen das Berufungsurteil legte die Partnerschaftsgesellschaft erfolgreich Revision ein. Der BGH stellte ebenfalls fest, dass das in $\S 18$ Abs. 1 Satz 3 Fall 1 BO BW enthaltene Verbot einer Beteiligung von Radiologen an einer Teilgemeinschaftspraxis, in der sich der Beitrag des Radiologen auf das Erbringen medizinisch-technischer Leistungen auf Veranlassung der übrigen Mitglieder beschränke, gegen das Grundrecht der Ärzte auf Berufsausübungsfreiheit aus Art. 12 Abs. 1 GG verstoße.

Berufsrechtliche Vorgaben der Teilgemeinschaftspraxis

Die berufsrechtlichen Regelungen für Ärzte sind in den Berufsordnungen der jeweiligen Landesärztekammern geregelt. Die Regelungen der Musterberufsordnung (MBO) sind demgegenüber für die Landesärztekammern nicht verbindlich und werden durch diese auch nicht immer einheitlich umgesetzt.

Die Möglichkeiten der beruflichen $\mathrm{Zu}$ sammenarbeit von Ärzten ist in $\S 18$ Abs. $1 \mathrm{MBO}$ geregelt und bestimmt, dass Ärzte sich zu Berufsausübungsgemeinschaften, Organisationsgemeinschaften, Kooperationsgemeinschaften und Praxisbünden zusammenschließen dürfen. Der Zusammenschluss von Ärzten zur gemeinsamen Berufsausübung darf auch zur Erbringung einzelnerLeistungen(=Teilgemeinschafts- 
praxis) erfolgen, sofern er nicht einer Umgehung des $\S 31 \mathrm{MBO}$ dient.

Nach § 31 MBO ist es Ärzten nicht gestattet, für die Zuweisung von Patienten oder Untersuchungsmaterial oder für die Verordnung oder den Bezug von Arznei- oder Hilfsmitteln oder Medizinprodukten ein Entgelt oder andere Vorteile zu fordern, sich oder Dritten zu versprechen oder gewähren zu lassen oder selbst zu versprechen oder zu gewähren. $\S 31 \mathrm{MBO}$ bezweckt die Gewährleistung unabhängiger ärztlicher Entscheidungen, welche frei von merkantilen Erwägungen sein sollen.

Eine Umgehung des $\S 31$ MBO liegt für den Fall der Gründung einer Teilgemeinschaftspraxis gemäß § 18 Abs. 1 Satz 3 MBO daher insbesondere dann vor, wenn

1. sich entweder der Beitrag des Arzts auf das Erbringen medizinisch-technischer Leistungen auf Veranlassung der übrigen Mitglieder einer Teilgemeinschaftspraxis beschränkt (Fall 1) oder

2. der Gewinn ohne Grund in einer Weise verteilt wird, der nicht dem Anteil der von ihnen persönlich erbrachten Leistungen entspricht (Fall 2). Hierbei ist zu beachten, dass die Anordnung einer Leistung, insbesondere aus den Bereichen der Labormedizin, der Pathologie und der bildgebenden Verfahren keinen Leistungsanteil im Sinne des Satzes 3 darstellt (vgl. $§ 18$ Abs. 1 Satz 4 MBO).

In seinen Entscheidungsgründen führt der BGH aus, dass die unterschiedslose Gewinnverteilung in einer Teilgemeinschaftspraxis unabhängig von der persönlich erbrachten Leistung des einzelnen Gesellschafters regelmäßig einen Hinweis auf eine Umgehung des $\S 31$ MBO darstelle und daher der 2 . Fall der Regelung in $\S$ 18 Abs. 1 Satz 3 MBO keinen rechtlichen Bedenken unterliege. Auch die Regelung des $\S 18$ Abs. 1 Satz 4 MBO, wonach „die Anordnung einer Leistung, insbesondere aus den Bereichen der Labormedizin, der Pathologie und der bildgebenden Verfahren keinen Leistungsanteil im Sinne des Satzes 3 darstellt" sei rechtlich nicht zu beanstanden, da dadurch verhindert werde, dass eine Zuweisung von Patienten oder Untersuchungsmaterial, für die bei nicht miteinander kooperierenden Ärzte keine Vorteile versprochen oder gewährt werden dürfe, innerhalb von beruflichen Kooperationen als zu vergütende Leistung behandelt werden könne.
Eingriff in die Berufsausübungsfreiheit nach Art. 12 Abs. 1 GG?

Unvereinbar mit der in Art. 12 Abs. 1 GG grundrechtlich verbürgten Berufsausübungsfreiheit und deshalb unwirksam ist dagegen nach Ansicht des BGH $\S 18$ Abs. 1 Satz 3 Fall 1 MBO, wonach eine Umgehung des $\S 31 \mathrm{MBO}$ und damit kein nach $\S 18$ MBO zulässiger Zusammenschluss zur gemeinsamen Ausübung des Arztberufs immer dann vorliegt, wenn sich der Beitrag des Arzts auf das Erbringen medizinisch-technischer Leistungen auf Veranlassung der übrigen Mitglieder einer Teilgemeinschaftspraxis beschränkt.

Damit fingiere die Berufsordnung eine Umgehung des $\S 31$ MBO mit der Folge eines Verbots einer Teilgemeinschaftspraxis auch in den Fällen, in denen eine unerlaubte Zuweisung nach den erkennbaren Umständen nicht vorliege. Somit stelle $\S 18$ Abs. 1 Satz 3 Fall 1 MBO nicht nur einen Eingriff in die Vertragsfreiheit der betroffenen Ärzte dar, sondern verletze auch deren durch Art. 12 Abs. 1 GG geschützte Berufsausübungsfreiheit.

In den Schutzbereich der Berufsausübungsfreiheit darf gemäß Art. 12 Abs. 1 Satz 2 GG nur auf der Grundlage einer gesetzlichen Regelung eingegriffen werden. Zudem muss der Eingriff verhältnismäßig sein. Hierbei hat das Gericht im Rahmen einer Abwägung danach zu fragen, ob der Grundsatz der Verhältnismäßigkeit eingehalten wurde. Ein Eingriff in Art. 12 Abs. 1 GG muss danach vernünftigen Zwecken des Allgemeinwohls dienen und darf den Berufstätigen nicht übermäßig oder unzumutbar treffen.

Nach Ansicht des BGH verstößt die in $\S 18$ Abs. 1 Satz 3 Fall 1 MBO enthaltene Regelung gegen den Grundsatz der Verhältnismäßigkeit. Zwar sei das dort statuierte abstrakte Verbot geeignet, dem Zweck zu dienen, nämlich die Unabhängigkeit ärztlicher Entscheidungen von merkantilen Erwägungen zu gewährleisten, jedoch stelle das Verbot weder ein erforderliches noch ein angemessenes Mittel dar, um den oben genannten Zweck zu erreichen.

Ein Eingriff in ein Grundrecht ist nur dann verhältnismäßig, wenn er erforderlich ist. Dies bedeutet, dass kein milderes und gleich wirksames Mittel zur Verfügung steht. Hierzu führt der BGH aus, dass bereits die in $\S 18$ Abs. 1 Satz 2, Satz 3 Fall 2 und Satz 4 MBO enthaltenen Regelungen dazu bestimmt und geeignet seien, dem genannten Zweck zu dienen. Diese sähen zum einen ein Verbot der Umgehung des $\S 31 \mathrm{MBO}$ und zum anderen im Grundsatz eine Gewinnverteilung vor, die dem Anteil der persönlich erbrachten ärztlichen Leistungen in einer Teilgemeinschaftspraxis entsprächen. Diese Regelungen seien daher geeignet, einen effektiven Schutz vor Formen der beruflichen Zusammenarbeit von Ärzten zu gewährleisten, bei denen die Unabhängigkeit der dabei zu treffenden ärztlichen Entscheidungen durch merkantile Erwägungen beeinträchtigt werde.

Der BGH weist daraufhin, dass Art. 12 Abs. 1 GG nicht nur für natürliche Personen, sondern auch juristische Personen gilt. Diese können sich gemäß Art. 19 Abs. 3 GG ebenfalls auf den Schutz des Grundrechts berufen, sofern dieses dem Wesen nach auch für juristische Personen anwendbar ist. Das bedeutet, dass der Eingriff auch die Gesellschaft selbst in ihrem Recht auf Berufsausübungsfreiheit beeinträchtigen muss. Dies hat vorliegend der BGH unter Hinweis auf die Beschränkung der Ärzte, die sich in der Gesellschaft zusammenschließen können, bejaht.

\section{Schutz vor}

\section{"Kickback-Konstellationen"}

Auch die angeblich besondere Anfälligkeit der medizinisch-technischen Überweisungsfächer für „Kickback-Konstellationen“ rechtfertigt nach Ansicht des BGH das in $\S 18$ Abs. 1 Satz 3 Fall 1 MBO statuierte Verbot nicht. Unter „Kickback-Leistungen" sind pauschale, vom wirtschaftlichen Gesamtgewinn der Teilgemeinschaftspraxis unabhängige Gewinnanteile zu verstehen, die den Anschein erwecken, eine bloße Bezahlung für Patientenüberweisungen darzustellen und somit gegen das Verbot der Zuweisung gegen Entgelt verstoßen. Aufgrund der Gefahr der Legalisierung der oben beschrieben „Kickback-Konstellationen“ durch den Umweg einer Teilgemeinschaftspraxis wurden die Vorgaben hinsichtlich des Zusammenschlusses von Ärzten restriktiv und zurückhaltend gehandhabt. Dem hält der BGH entgegen, dass der Zuweisung von Patienten gegen Entgelt durch Ärzte teils effektiver durch die Prüfung der gesellschaftsrechtlichen Konstruktion im Einzelfall entgegengetreten werden könne. Dies habe den Vorteil, dass das Kooperationsverhalten der betroffenen Ärzte nicht von vornherein erheblich eingeschränkt bzw. gänzlich unterbunden wer- 
de. Der BGH argumentiert in seiner Entscheidung, dass die Ärztekammern über verhältnismäßigere Kontrollmechanismen verfügen würden und sich die Gesellschaftverträge vorlegen lassen könnten, wie es auch $\S 18$ Abs. 1 Satz 5 MBO normiere.

\section{Kritische Betrachtung des BGH-Urteils}

Im Ergebnis hält der BGH ausschließlich die Regelung in $\S 18$ Abs. 1 Satz 3 Fall 1 BO BW, wonach eine Umgehung des $\S 31$ der BO BW und damit kein zulässiger Zusammenschluss vorliegt, „wenn sich der Beitrag des Arzts auf das Erbringen medizinisch-technischer Leistungen auf Veranlassung der übrigen Mitglieder einer Teilberufsausübungsgemeinschaft beschränkt“, mit Art. 12 Abs. 1 GG für unvereinbar.

Wie die Vorinstanz, das OLG Karlsruhe festgestellt hat, beschränkte sich vorliegend der Beitrag der an der Teilgemeinschaftspraxis beteiligten Radiologen auf die Erbringung bestimmter medizinischtechnischer Leistungen - nämlich auf Knochendichtemessungen (Osteodensitometrie) - auf Anordnung der übrigen Gesellschafter (OLG Karlsruhe, Urt. v. 27.06.2012, Az.: 6 U 15-11). Diese Form der Zusammenarbeit hielt des OLG für unzulässig, da sich aus $\S 18$ Abs. 1 Satz 3 Fall 1 BO BW ergebe, „dass eine Teilberufsausübungsgemeinschaft unter Einbeziehung von Angehörigen sogenannter Methodenfächer - etwa von Radiologen - zulässig ist, wenn ihr Beitrag nicht auf die Erbringung medizinisch-technischer Leistungen auf Veranlassung der übrigen Gesellschafter beschränkt ist, sondern sie im Rahmen der Teilberufsausübungsgemeinschaft darüber hinaus weitere Leistungen erbringen."

Diese Auslegung der Regelung in $\S 18$ Abs. 1 Satz 3 Fall 1 BO BW durch das OLG Karlsruhe ist jedoch mit der Intention des Normgebers nicht zu vereinbaren. Die Bundesärztekammer und die Landesärztekammern, die die Regelung in ihre Berufsordnungen übernommen haben, wollten mit der Vorgabe, dass sich der Beitrag des Arzts nicht „auf das Erbringen medizinisch-technischer Leistungen auf Veranlassung der übrigen Mitglieder einer Teilberufsausübungsgemeinschaft beschränken darf“ erreichen, dass die in einer Teilgemeinschaftspraxis erbrachten medizinisch-technischen Leistungen gemeinsam von den Gesellschaftern erbracht werden. Sinn und Zweck der Teilgemeinschaftspraxis ist es nämlich, dass die Berufsausübung der Ärzte darauf gerichtet ist, die betreffenden ärztlichen Leistungen gemeinsam zu erbringen. Voraussetzung für eine gemeinsame Berufsausübung in einer Teilgemeinschaftspraxis ist daher, dass die Gesellschafter fachlich und tatsächlich in der Lage sind, Teile einer einheitlichen ärztlichen Leistung gemeinsam zu erbringen. Mit anderen Worten erlaubt $\S 18$ Abs. 1 Satz 3 BO BW „die Bildung von Berufsausübungsgemeinschaften zur Übernahme spezifischer, auf die Erbringung bestimmter Leistungen bezogener Behandlungsaufträge“ (vgl. die Begründung zum Gesetzentwurf des VÄndG vom 30.08.2006, BTDrucks. 16/2474, S. 31).

Die Bundesärztekammer hat hierauf in ihrer Publikation „Niederlassung und berufliche Kooperation“ (DÄBl. 2006, A-801) zur Erläuterung der neuen Vorschriften in der MBO für den Bereich medizinischtechnischer Leistungen hingewiesen:

„Dabei ist es zulässig, dass innerhalb der Teilgemeinschaftspraxis die Leistungserbringung zwischen den Beteiligten aufgegliedert wird (z.B. die arbeitsteilige Trennung von Untersuchungsleistung und Befundung, insbesondere bei bildgebender Diagnostik). Dieses gilt auch für überörtliche Teilgemeinschaftspraxen, bei denen eine Leistungsaufteilung $z$.B. auch unter Einsatz telemedizinischer Verfahren denkbar ist.“

Hieraus folgt, dass Gegenstand einer Teilgemeinschaftspraxis, entgegen der Ansicht des OLG Karlsruhe, auch medizinisch-technische Leistungen sein können, die der Radiologe auf Veranlassung der übrigen Gesellschafter erbringt und dass es nicht erforderlich ist, dass dieser im Rahmen der Teilgemeinschaftspraxis darüber hinaus weitere Leistungen erbringt. Voraussetzung ist allerdings, dass der Radiologe die Leistung gemeinsam mit den anderen Gesellschaftern vor Ort durchführt (vgl. Wigge / Kaiser / Fischer / Loose, MedR 2010, 700, 708).

Insoweit ist es, entgegen der Ansicht des $\mathrm{BGH}$, keine unzulässige Einschränkung der Berufsfreiheit nach Art. 12 Abs. 1 GG, wenn in $\S 18$ Abs. 1 Satz 3 Fall 1 BO BW gefordert wird, dass die medizinischtechnischen Leistungen vom Radiologen nicht allein („auf Veranlassung“), sondern gemeinschaftlich mit den beteiligten Ärzten innerhalb der Teilgemeinschaftspraxis erbracht werden. Zwar ist zuzugeben, dass die Formulierung in $\S 18$ Abs. 1 Satz 3 Fall 1 BO BW etwas umständlich ist, jedoch soll hierdurch zum Ausdruck gebracht werden, dass es für die Zulässigkeit der Erbringung medizinisch-technischer Leistungen in einer Teilgemeinschaftspraxis nicht ausreichend ist, dass ein Arzt eines therapieorientierten Fachgebietes die radiologische Untersuchungsleistung nur „veranlasst“, sondern sich an dieser aktiv beteiligt.

Beispiele für zulässige Formen einer derartigen gemeinsamen Leistungserbringung von radiologischen Leistungen in einer Teilgemeinschaftspraxis sind etwa:

- die computertomografisch gesteuerte Infiltration der Zwischenwirbelgelenke zwischen Radiologe und Orthopäde,

- MRT-Leistungen des Herzens zwischen Radiologen und Kardiologe,

- Mammografieleistungen zwischen Radiologen und Gynäkologen.

Die vorgenannten Beispiele machen deutlich, dass dem im Weiterbildungsrecht verankerten Verbot der fachgebietsfremden Tätigkeit in diesem Zusammenhang eine wesentliche Bedeutung zukommt und eine Verletzung dieses Verbotes zugleich ein wichtiges Indiz für einen Gestaltungsmissbrauch bei der Beurteilung einer Teilgemeinschaftspraxis zwischen Radiologen und anderen Fachgruppen zukommt. Im Bereich der bildgebenden Diagnostik sind therapeutisch tätige Facharztgruppen weiterbildungsrechtlich häufig zur Mitbefundung und/oder Befundauswertung berechtigt, sodass in diesem Teil der Leistung eine Möglichkeit der Zusammenarbeit mit dem Radiologen besteht (Wigge, NZS 2007, 393, 396). Im Bereich der Magnetresonanztomografie vermittelt die auf der Grundlage des in Abschnitt B des geltenden Weiterbildungsrechts erteilte Berechtigung zur Führung der Zusatzbezeichnung „MRT- fachgebunden“ insbesondere Orthopäden und Kardiologen eine entsprechende Fachkunde (vgl. Wigge / Kaiser / Fischer / Loose, MedR 2010, 700, 702).

Die gebührenrechtliche Zulässigkeit einer gemeinsamen Leistungserbringung nach der GOÄ stellt einen weiteren Gesichtspunkt dar. Entscheidend für die berufsrechtliche Zulässigkeit der Teilgemeinschaftspraxis ist jedoch, dass die beteilig- 
ten Ärzte auch ärztliche Leistungen erbringen, die im Rahmen der Gesamtbehandlung bzw. der Diagnostik als Teilbeitrag angesehen werden können (vgl. Wigge/Kaiser/Fischer/Loose, MedR 2010, 700, 708; Wigge, NZS 2007, 393, 396). Die Gründung einer Teilgemeinschaftspraxis ist daher zulässig, wenn eine gemeinsame Erbringung der einzelnen diagnostischen Schritte möglich ist und eine Beteiligung an der Durchführung der Leistung seitens der betreffenden Ärzte erfolgt. Auch hier wird allerdings vorausgesetzt, dass die in der Gesellschaft tätigen Ärzte während der Durchführung der Untersuchung und der anschließenden Befundung am Ort der radiologischen Leistung anwesend sind. Bei der Gewinnverteilung stellen die berufsrechtlichen Bestimmungen auf den jeweiligen Anteil der persönlich erbrachten Leistungen ab, sodass eine tatsächliche Berufsausübung erforderlich ist und nicht lediglich virtuelle Praxisbünde ausreichend sind (vgl. Wigge/Kaiser/Fischer/Loose, MedR 2010, 700, 708).

Insoweit stellt die Erbringung von medizinisch-technischen Leistungen auf Veranlassung der übrigen Gesellschafter durch den Radiologen allein keine „gemeinsame Berufsausübung“ im Sinne einer Teilgemeinschaftspraxis dar. Dieses Erfordernis der arbeitsteiligen Leistungserbringung kann, entgegen der Auffassung des BGH, auch nicht durch Kontrollmechanismen der Ärztekammern ersetzt werden, da es sich um das entscheidende Wesensmerkmal einer Teilgemeinschaftspraxis handelt. Anders als der BGH meint, wird durch die Regelung in $\S 18$ Abs. 1 Satz 3 Alt. 1 BO BW auch nicht eine Beteiligung von Radiologen und anderen methodendefinierten Fachgebieten an Teilgemeinschaftspraxen mit anderen Ärzten prinzipiell ausgeschlossen. Vielmehr verlangt die Vorschrift für eine zulässige Form der Zusammenarbeit in einer Teilgemeinschaftspraxis eine gemeinsame Leistungserbringung im Bereich medizinischtechnischer Leistungen. Andernfalls ist bereits die Gründung der Gemeinschaftspraxis berufsrechtlich unzulässig und damit der Zusammenschluss aller beteiligten Ärzte.

Durch den Wegfall der Regelung in $\S 18$ Abs. 1 Satz 3 Alt. 1 BO BW wird es möglich sein, dass die therapeutisch tätigen $\mathrm{Ge}$ sellschafter einer Teilgemeinschaftspraxis dem Radiologen in der Gesellschaft Patienten zuweisen, die dieser dann alleine untersucht. Damit wird der Sinn und
Zweck der Teilgemeinschaftspraxis, nämlich einen spezifischen, auf die Erbringung bestimmter Leistungen bezogenen Behandlungsauftrag gemeinsam zu erfüllen, konterkariert. Die therapeutisch tätigen Ärzte in der Teilgemeinschaftspraxis sind damit an der Leistungserbringung nicht mehr zwingend $\mathrm{zu}$ beteiligen und erhalten eine gesellschaftsrechtliche Beteiligung an den Einnahmen des Radiologen. Gerade dies will jedoch $\S 31$ der Berufsordnung ausdrücklich verhindern.

Der Entscheidung des BGH und der von ihm geäußerten Auffassung, wonach die Regelung in $\S 18$ Abs. 1 Satz 3 Fall 1 BO BW mit der in Art. 12 Abs. 1 GG grundrechtlich verbürgten Berufsausübungsfreiheit unvereinbar und deshalb unwirksam ist, kann daher nicht gefolgt werden.

\section{Auswirkungen auf die Teilgemein-} schaftspraxis im Vertragsarztrecht Die Entscheidung des BGH beschäftigt sich ausdrücklich nur mit den Vorgaben für die Teilgemeinschaftspraxis in $\S 18$ Abs. 1 Satz 3 der Berufsordnung der Landesärztekammer Baden-Württemberg (BO BW). Die bisherige Formulierung des $\S 18$ Abs. 1 Satz 3 der BO BW findet sich in dieser Form - mit Ausnahme von Bayern, Berlin, Hamburg und Rheinland-Pfalz - in den meisten Berufsordnungen der übrigen Landesärztekammern sowie $\S 18$ der Musterberufsordnung der Bundesärztekammer (MBO). Die Landesärztekammer Baden-Württemberg hat nun, als Reaktion auf das Urteil des BGH, durch eine Änderung des $\S 18$ Abs. 1 Satz 3 BO BW, das Verbot der Zusammenarbeit einer Teilgemeinschaftspraxis mit Radiologen unter den dort genannten Voraussetzungen außer Kraft gesetzt. Ob dies auch in den übrigen Landesärztekammern erfolgen wird, bleibt abzuwarten.

Keine Aussage hat der BGH demgegenüber zu der gleichlautenden Bestimmung in $\S$ 33 Abs. 2 Satz 3 Ärzte-ZV getroffen. Ursprünglich sah diese Regelung noch ein ausnahmsloses Verbot der Teilgemeinschaftspraxis für Radiologen und andere überweisungsabhängigen Vertragsärzte im Sinne von $\S 14$ Abs. 4 BMV-Ä vor. Mit dem Inkrafttreten des VÄndG vom 22.12.2006 wurde in $\S 33$ Abs. 2 Satz 3 Ärzte-ZV folgende Regelung für die Teilgemeinschaftspraxis aufgenommen:

"Die gemeinsame Berufsausübung bezogen auf einzelne Leistungen ist zulässig, sofern diese Berufsausübungsgemeinschaft nicht zur Erbringung überweisungsgebundener medizinisch-technischer Leistungen mit überweisungsberechtigten Leistungserbringern gebildet wird.“

Damit war im Vertragsarztrecht ein $\mathrm{Zu}-$ sammenschluss von oder mit überweisungsabhängigen Facharztgruppen im Rahmen einer Teilgemeinschaftspraxis nach § 13 Abs. 4 BMV-Ä zunächst ausnahmslos untersagt. Der Gesetzgeber begründete dieses Verbot damit, dass „sog. Kickback-Konstellationen“, bei denen ein Arzt eines therapieorientierten Fachgebietes (z.B. Gynäkologe) eine Berufsausübungsgemeinschaft mit einem Arzt eines Methodenfaches (z. B. Labor) eingeht, um das berufsrechtliche Verbot der $\mathrm{Zu}$ weisung gegen Entgelt zu unterlaufen, nicht erlaubt werden sollten.

Dieses generelle Verbot wurde im Rahmen des GKV-VStG ab dem 01.01.2012 gelockert, in dem der Gesetzgeber die Formulierungen in $\S 18$ Abs. 1 Satz 3 MBO weitgehend übernommen hat. Auch in $\S$ 33 Abs. 2 Satz 3 Ärzte-ZV findet sich seitdem die vom BGH als mit Art. 12 Abs. 1 GG für unvereinbar erklärte Vorgabe, dass eine Umgehung des in $\S 73$ Abs. 7 SGB V für den Bereich des Vertragsarztrechts geregelten Zuweisungsverbots insbesondere vorliegt, „wenn sich der Beitrag des Arztes auf das Erbringen medizinisch-technischer Leistungen auf Veranlassung der übrigen Mitglieder einer Berufsausübungsgemeinschaft beschränkt.“

Damit besteht auch im Vertragsarztrecht das Verbot, dass sich ein Radiologe nicht an einer Teilgemeinschaftspraxis beteiligen darf, wenn sich sein Leistungsanteil auf das Erbringen medizinisch-technischer Leistungen auf Veranlassung der übrigen Ärzte der Teilgemeinschaftspraxis beschränkt. Dieses Verbot in $\S 33$ Abs. 2 Satz 3 Ärzte-ZV hat auch nach der Entscheidung des BGH im Vertragsarztrecht weiterhin Gültigkeit. Hierbei ist zu berücksichtigen, dass es sich bei der Ärzte$\mathrm{ZV}$, anders als bei den Berufsordnungen der Ärztekammern, um ein förmliches Bundesgesetz handelt, über dessen Verfassungswidrigkeit nur das Bundesverfassungsgericht nach Art. 100 Abs. 1 GG entscheiden kann(vgl. BSG, Urt. v. 16.07.2003, Az.: B 6 KA 49/02 R).

$\mathrm{Zu}$ beachten ist auch, dass im Vertragsarztrecht über $\S 33$ Abs. 2 Satz 2 Ärzte-ZV 
i.V.m. § 73 Abs. 7 Satz 2 SGB V die Regelung des $\S 128$ Absatz 2 Satz 3 SGB V entsprechend gilt. Nach dieser Vorschrift, die durch den Verweis auch für die Zusammenarbeit von Vertragsärzten untereinander Geltung beansprucht, werden „Einkünfte aus Beteiligungen an Unternehmen von Leistungserbringern, die Vertragsärzte durch ihr Verordnungs- oder Zuweisungsverhalten selbst maßgeblich beeinflussen“ als unzulässige Zuwendungen angesehen. Auch nach dieser Vorschrift ist eine Teilgemeinschaftspraxis daher nicht genehmigungsfähig, in der therapeutisch tätige Ärzte und Radiologen zusammengeschlossen sind und die therapeutisch tätigen Ärzte dem Radiologen in maßgeblichem Umfang Untersuchungsleistungen zuweisen, ohne dass diese selbst an der Erbringung dieser Leistungen beteiligt sind.

Die Auswirkungen der BGH-Entscheidung auf das Vertragsarztrecht sind daher nicht abschließend geklärt, da die Rechtslage hier anders gelagert ist. Es sollte jedoch möglichst umgehend eine Klarstellung erfolgen, da ein Verstoß gegen das Zuweisungsverbot in $\S 73$ Abs. 7 Satz 2 SGB V und $\S 33$ Abs. 2 Satz 2 Ärzte-ZV zur Rechtswidrigkeit der Teilgemeinschaftspraxis und damit zu erheblichen vertragsarztrechtlichen Konsequenzen bis zur Rückforderung der Honorareinnahmen durch die Kassenärztliche Vereinigung führen kann.

\section{Fazit}

\section{$\nabla$}

Den Ausführungen in dem Urteil des BGH kann im Ergebnis nicht zugestimmt werden. Die Entscheidung macht vielmehr deutlich, dass der BGH das Grundprinzip der Teilgemeinschaftspraxis, als eine Form der gemeinsamen ärztlichen Berufsausübung „zur Übernahme spezifischer, auf die Erbringung bestimmter Leistungen bezogener Behandlungsaufträge“ (vgl. die Begründung zum Gesetzentwurf des VÄndG vom 30.08.2006, BTDrucks. 16/2474, S. 31) nicht nachvollzogen hat.

Zudem ist die Ansicht des BGH nicht Zutreffend, dass die Verhinderung von „Kickback-Konstellationen" bereits durch die übrigen Vorgaben des $\S 18$ Abs. 1 Satz 3 MBO erreicht werden kann, denn die Art der Gewinnverteilung allein kann missbräuchliche Vertragsgestaltungen nicht verhindern. Ist nämlich z.B. der Orthopä- de oder Kardiologe an der Erbringung von MRT-Untersuchungen des Radiologen überhaupt nicht beteiligt, steht ihm keinerlei Anteil an den in der Teilgemeinschaftspraxis erwirtschafteten Gewinnen für diese Leistung zu. Die Teilgemeinschaftspraxis verstößt in diesem Fall gegen $\S 31$ der Berufsordnung und ist daher berufsrechtlich zu untersagen. Die Regelung in $\S 18$ Abs. 1 Satz 4 MBO, wonach die Anordnung einer Leistung, insbesondere aus den Bereichen der Labormedizin, der Pathologie und der bildgebenden Verfahren, keinen Leistungsanteil darstellt, verdeutlicht zwar, dass die Zuweisung eines Gesellschafters der Teilgemeinschaftspraxis an den Radiologen keine ausreichende Beteiligung an der Leistungserbringung begründet. Die Vorschrift verhindert jedoch nicht, dass eine gesellschaftsrechtliche Beteiligung der Mitgesellschafter an den Leistungen des Radiologen stattfindet, die dieser aufgrund von Überweisungen durch Ärzte erbringt, die keine Gesellschafter der Teilgemeinschaftspraxis sind.

Ebenso ist die Rechtslage im Vertragsarztrecht zu beurteilen. Um eine Einheitlichkeit von Berufs- und Vertragsarztrecht im Rahmen der ärztlichen Berufsausübung in Teilgemeinschaftspraxen zu erreichen, sollten die Bundesärztekammer und der Gesetzgeber die Regelung in $\S 18$ Abs. 1 Satz 3 MBO und § 33 Abs. 2 Ärzte-ZV überarbeiten, jedoch klarstellen, dass Voraussetzung für eine Teilgemeinschaftspraxis die berufs-, weiterbildungs- und vertragsarztrechtlich zulässige Übernahme eines gemeinsamen Untersuchungs- bzw. Behandlungsauftrags der beteiligten Ärzte ist. Andernfalls degeneriert die Organisationsform der Teilgemeinschaftspraxis tatsächlich zu einem reinen „KickbackModell“.

Prof. Dr. Peter Wigge

Rechtsanwalt, Fachanwalt für Medizinrecht

Stefanie Broß

Rechtsanwältin

Rechtsanwälte Wigge

Scharnhorststr. 40

48151 Münster

Telefon: (0251) 53595-0

Telefax: (0251) 53595-99

E-Mail: kanzlei@ra-wigge.de

www.ra-wigge.de 